\title{
Continuous positive airway pressure therapy in non-sleepy patients with obstructive sleep apnea: results of a meta-analysis
}

\author{
Dongmei Zhang, Jinmei Luo, Yixian Qiao, Yi Xiao \\ Department of Respiratory Medicine, Peking Union Medical College Hospital, Peking Union Medical College, Chinese Academy of Medical \\ Sciences, Beijing 100730, China \\ Contributions: (I) Conception and design: Y Xiao, J Luo; (II) Administrative support: None; (III) Provision of study materials or patients: None; \\ (IV) Collection and assembly of data: D Zhang, Y Qiao; (V) Data analysis and interpretation: Y Xiao, J Luo, D Zhang; (VI) Manuscript writing: All \\ authors; (VII) Final approval of manuscript: All authors. \\ Correspondence to: Dr. Yi Xiao. Department of Respiratory Medicine, Peking Union Medical College Hospital, Peking Union Medical College, \\ Chinese Academy of Medical Sciences, Beijing 100730, China. Email: xiaoyipumch@sina.com.
}

Background: Although continuous positive airway pressure (CPAP) has become the first line of therapy for obstructive sleep apnea (OSA), it remains controversial whether non-sleepy patients could benefit from CPAP treatment.

Methods: We searched the online databases Medline, Embase, the Cochrane library and the Cochrane Central Registry of Controlled Trials to select eligible control trials, including non-sleepy OSA patients and those patients treated by CPAP or either sham CPAP or no CPAP.

Results: Seven eligible studies (1,541 patients) were included. The pooled estimates of the mean changes after CPAP treatment for the systolic blood pressure (SBP) and diastolic blood pressure (DBP) were $-0.51 \mathrm{mmHg}(95 \% \mathrm{CI},-3.39$ to $2.38 \mathrm{mmHg}$; $\mathrm{P}=0.73)$ and $-0.92 \mathrm{mmHg}$ (95\% CI, -1.39 to $-0.46 \mathrm{mmHg}$; $\mathrm{P}<0.001)$, respectively. CPAP should not improve subjective sleepiness in the minimally symptomatic OSA patients, as the change in the Epworth sleepiness scale (ESS) was -0.51 (95\% CI, -1.68 to $0.67 ; \mathrm{P}=0.397$ ). However, CPAP can effectively reduce AHI or ODI by 15.57 events/h (95\% CI, -29.32 to $-1.82 ; \mathrm{P}=0.026$ ) compared to controls. However, the risk of cardiovascular events did not significantly decrease [odds ratio (OR), 0.80; 95\% CI, 0.50 to 1.26 ; $\mathrm{P}=0.332$ ] in the end.

Conclusions: CPAP treatment can reduce OSA severity in non-sleepy patients and minutely reduce the DBP, but CPAP seems to have no overall beneficial effects on subjective sleepiness, SBP, or cardiovascular risk.

Keywords: Sleep apnea; obstructive/complications/therapy; continuous positive airway pressure/method (CPAP/ method); hypertension; cardiovascular diseases

Submitted May 12, 2016. Accepted for publication Aug 15, 2016.

doi: $10.21037 /$ jtd.2016.09.40

View this article at: http://dx.doi.org/10.21037/jtd.2016.09.40

\section{Introduction}

Obstructive sleep apnea (OSA) is a common disease with an estimated prevalence of approximately $22 \%$ in men and $17 \%$ in women (1). This disorder is characterized by recurrent collapse of the upper airway during sleep, resulting in repetitive asphyxia and arousal from sleep. These fragmentations of sleep lead to daytime sleepiness, decreased quality of life, and increased risk of traffic accidents and industrial injuries. By increasing the intraluminal pressure in the pharynx, continuous positive airway pressure (CPAP) treatment is effective for all degrees of OSA, and CPAP has been shown to reduce excessive sleepiness and cardiovascular events.

The prevalence of non-sleepy OSA [with an Epworth sleepiness scale (ESS) score $\leq 10$ and whose sleepiness 
does not disturb social or work activities] among middleaged adults has been shown to be as high as $30 \%(2,3)$. The management of non-sleepy subjects constitutes a major challenge. Whether CPAP should be strongly recommended in non-sleepy OSA patients with a high apnea-hypopnea index (AHI), especially where there is an association with cardiovascular disorders, is still a matter of debate. The findings from randomized controlled trials (RCTs) have been controversial. A meta-analysis including four RCTs shows that CPAP can reduce OSA severity and sleepiness, but CPAP cannot reduce blood pressure in minimally symptomatic OSA patients, except in those patients with optimal adherence to CPAP (4). Whether non-sleepy patients can benefit from CPAP treatment in other respects, such as reducing cardiovascular endpoint risk, is still unknown. As only a limited number of such patients have been included in each trial, we performed a meta-analysis to answer this clinically and epidemiologically important question.

\section{Methods}

This systematic review and meta-analysis adheres to the Preferred Reporting Items for Systematic Review and Metaanalyses (PRISMA) statement for reporting on systematic reviews (5). Additionally, we conducted the meta-analysis following the Cochrane Handbook for Systematic Reviews of Interventions.

\section{Data sources and literature searches}

We searched four online databases: Medline, Embase, the Cochrane Library and the Cochrane Central Registry of Controlled Trials from January 1, 1996 to April 1, 2016. Keywords used to identify RCTs of CPAP in the treatment of OSA were (apnoea.af or apnea.af or hypopnoea.af or hypopnea.af) and (CPAP.af or continuous positive airway pressure.af or positive airways pressure.af or positive pressure. af) and (randomized controlled trial.pt or. ti or clinical trial. pt or .ti) and (minimally symptomatic.af or nonsleepy.af or asymptomatic.af). Suffixes indicated search fields used in Medline $(. a f=$ all fields, $. p t=$ publication type and $. t i=$ title $)$. Reference lists of all identified trials and review articles were also screened for relevant trials. Clinical trial registries were searched for unpublished trials (supplementary: search details).

\section{Inclusion criteria}

Trials must include patients diagnosed with minimally symptomatic, asymptomatic or non-sleepy OSA, and trials must group patients into a CPAP treatment group and a control group [sham-CPAP or no CPAP (standard care)]. Studies that were RCTs or prospective case control trials (CCTs) with treatment groups of at least 4 weeks of CPAP therapy undertaken on adult patients were included. Two investigators (D Zhang and J Luo) independently applied the following selection rules to the studies identified in the systematic search.

\section{Validity assessment}

Before abstracting data, two investigators independently assessed the studies using the Jadad scale, which is a validated scale designed to measure trial quality. This scale ranges from 0 to 5 by scoring the randomization, double blinding and inclusion of detailed data on dropouts and withdrawals. There were no disagreements between the investigators on the Jadad scores of included trials.

\section{Data collection}

A list of the required variables for analysis was included in the protocol before data collection. Baseline and follow-up data on ESS and AHI [or oxygen desaturation index (ODI)] were desirable, along with age, gender, body mass index (BMI), blood pressure and the number of patients who used antihypertensive drugs. CPAP treatment usage (mean hours used per night) during follow-up was collected for patients randomized to the CPAP group. Systolic blood pressure (SBP) and diastolic blood pressure (DBP) measurements from baseline and follow-up were required along with treatment allocation. The cardiovascular events included nonfatal myocardial infarction, revascularization, stroke, transient ischemic attack, hospitalization for unstable angina or arrhythmia, heart failure, cardiovascular death and allcause mortality. The data of cardiovascular events were also needed in all the follow-up visitors. If we could not contact the authors, we relied on data reported in previous metaanalyses.

\section{Data synthesis and analysis}

STATA 12.0 (STATACorp, College Station, Texas, USA) was used for all statistical analysis, using the metan, metareg, metaninf and metabias commands to generate pooled estimates and plots. The mean differences in treatment responses between CPAP and control groups 


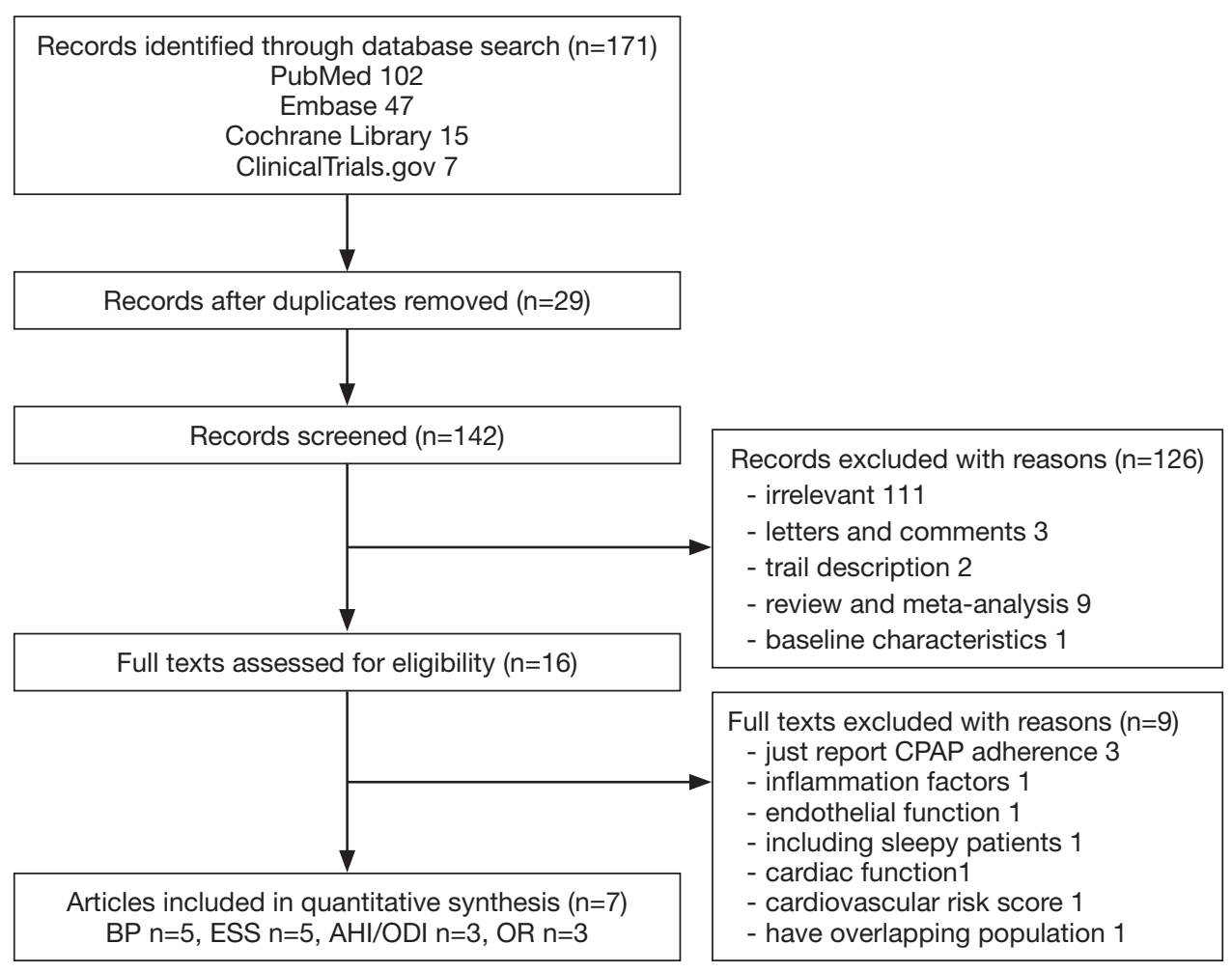

Figure 1 Trial inclusion flow chart.

and the standard error of that difference from individual studies were meta-analyzed (METAN). Pooled estimates of treatment responses were calculated with random effects model assumptions. Heterogeneity in treatment effects between trials was tested by using the $\mathrm{I}^{2}$ analysis. Summary estimates were also calculated excluding each study sequentially to measure the influence of individual study effects on the pooled estimate (METANINF). Publication bias was evaluated visually using the funnel plot and statistically (METABIAS) using Egger's and Begg's tests. The outcome measures were the mean differences between the treatment and control groups in the change in blood pressure, AHI, ESS and risk of cardiovascular diseases from baseline to the end of the trials.

\section{Results}

\section{Identification and description of included studies}

The systematic literature search identified 171 articles. After applying the exclusion criteria, both authors agreed that 7 articles were eligible with 1,541 patients in total. The reasons for excluding the other 164 studies are shown in Figure 1. One study about the effect of CPAP on cardiovascular biomarkers in minimally symptomatic OSA patients (6) was excluded because the study only involved 13 patients, and among them, 2 patients had ESS values greater than 10 . One article (7) about the 1-year effects of CPAP on hypertension was excluded because more exhaustive results were published in another article (8). The MOSAIC (Multicenter Obstructive Sleep Apnea Interventional Cardiovascular) trial reported many aspects of the effects of CPAP in minimally symptomatic OSA, such as the vascular function (9), cardiac function (10), calculated cardiovascular risk (11), inflammation factors (12), subjective sleepiness measured by the maintenance of wakefulness test and quality of life assessments (13); but we did not find enough data published in other trials, so we could not include these papers in our meta-analysis. Of the remaining articles, 5 studies $(8,14-17)$ recorded the change in BP and ESS, 3 studies $(14,15,17)$ reported the change of AHI or ODI and 3 studies $(8,18,19)$ reported the cardiovascular events in long term follow-up.

The detailed design characteristics and Jadad score judging the bias risk of the included studies are summarized in Table 1, and the patients' baseline characteristics are summarized in Table 2. The article Turnbull et al. [2014] (18) was a subset 


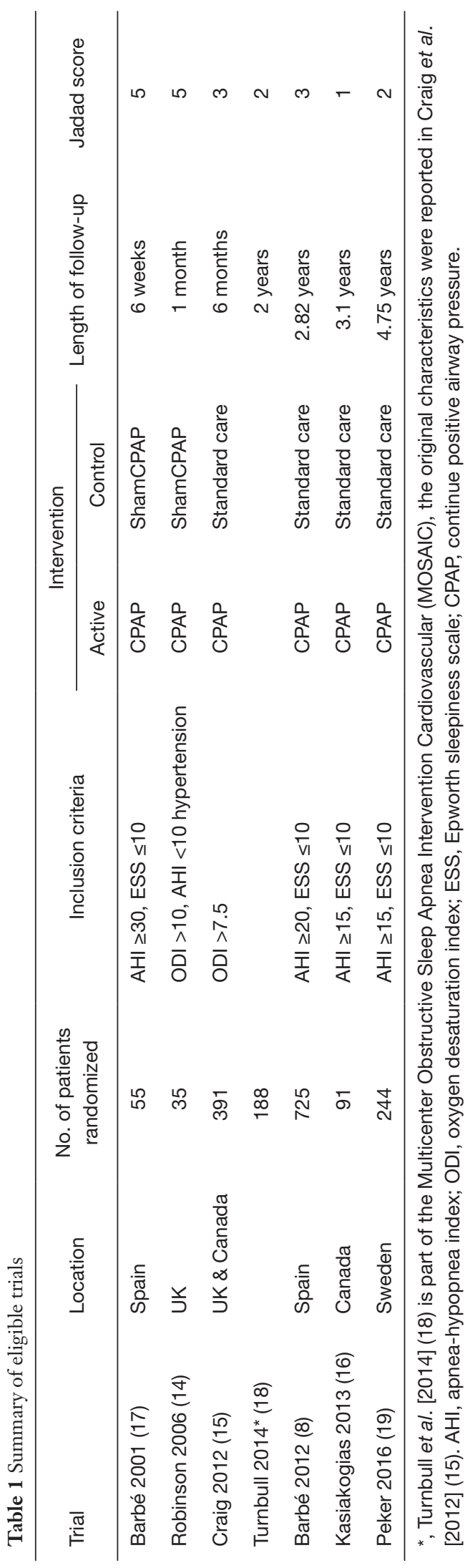

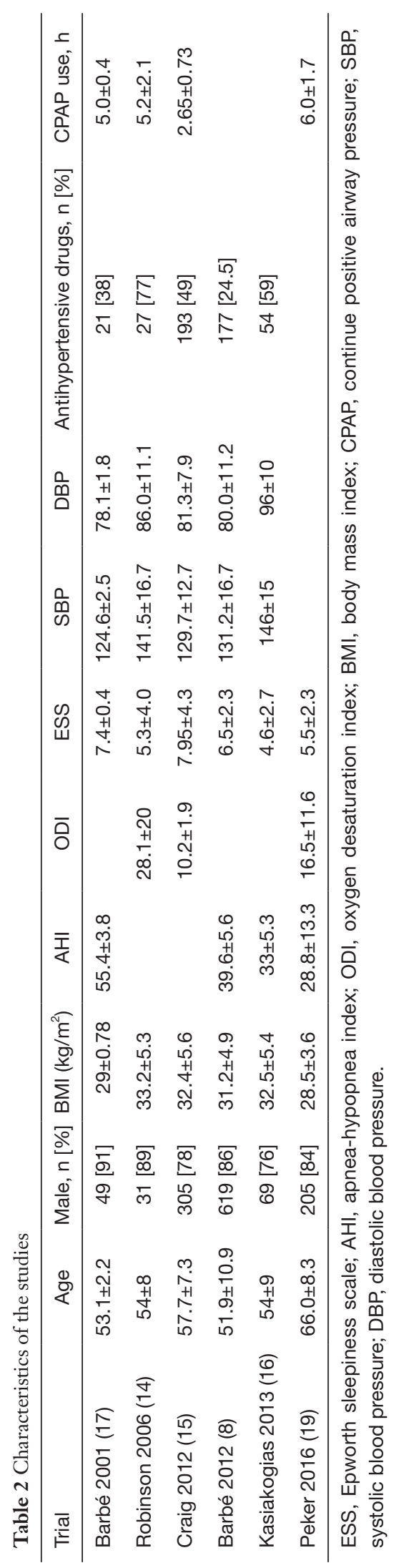




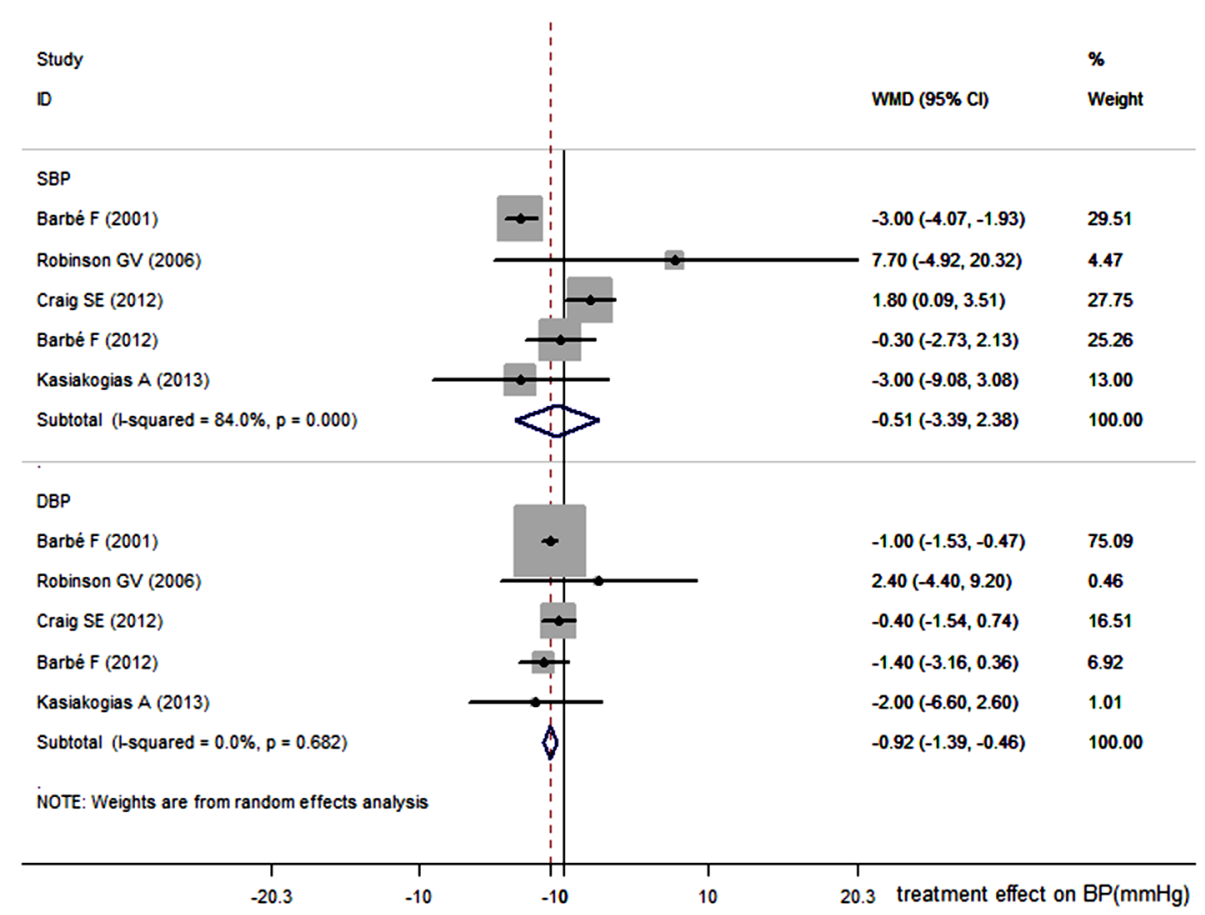

Figure 2 Effect of CPAP on systolic and diastolic blood pressures (SBP and DBP). CPAP, continuous positive airway pressure; SBP, systolic blood pressure; DBP, diastolic blood pressure.

of the MOSAIC trial, and 188 patients of the original trial were followed for 2 years to record the cardiovascular events. Because the original characteristics were reported in Craig et al. [2012] (15), we did not repeat the characteristics of the subset article. Only the first period data of the crossover study by Robinson et al. (14) were used. Two small studies $(14,17)$ used the sham-CPAP as the control and had a shorter follow-up period, while the remaining trials $(8,15,16,19)$ compared the CPAP with no CPAP and had a follow-up period of at least 6 months. The quality of the studies varied substantially, as two small studies $(14,17)$ had a Jadad score of 5 , two studies $(8,15)$ had a Jadad score of 3 and the other two studies $(16,19)$ had Jadad scores of 2 and 1 , respectively. Even with low Jadad scores, the results of the studies were cautiously assessed and considered comprehensive and reliable, so we did not exclude them in our analysis. The common reason for the deduction score was the lack of double blinding because the control groups only used pills or conservative therapy. The OSA severity range was wide, and the average AHI or ODI varied from 10.2 to 55.4 events/h in these trials. Because ODI and AHI are relatively similar, and for pragmatic reasons were assumed to be the same, we used ODI if AHI was unavailable. All studies regarded patients with an ESS $\leq 10$ as non-sleepy; apart from the MOSAIC trial, the mean ESS was 7.95, allowing the clinician to decide whether a patient was minimally symptomatic by a pragmatic approach.

\section{Analyses}

The primary outcomes were the absolute change in SBP and DBP between baseline and follow-up. Secondary outcomes were changes in ESS, AHI (ODI) and the cardiovascular risk. The absolute change in SBP and DBP between baseline and follow-up in Figure 2 shows that there was a slight decrease both in SBP and DBP of $-0.51 \mathrm{mmHg}(95 \%$ CI, -3.39 to $2.38 \mathrm{mmHg}$; $\mathrm{P}=0.73)$ and $-0.92 \mathrm{mmHg}(95 \%$ CI, -1.39 to $-0.46 \mathrm{mmHg} ; \mathrm{P}<0.001$ ), respectively in the CPAP group, compared with the control group. Significant heterogeneity was present among studies $\left(\mathrm{I}^{2}=84 \%, \mathrm{P}<0.001\right)$ in SBP. However, the summary estimates show that CPAP therapy could not reduce the SBP. The CPAP treatment can reduce DBP by $0.92 \mathrm{mmHg}$ compared with the control.

As shown in Figure 3, CPAP may not improve subjective sleepiness assessed by the ESS scale compared with the control group as the difference was -0.51 (95\% CI, -1.68 


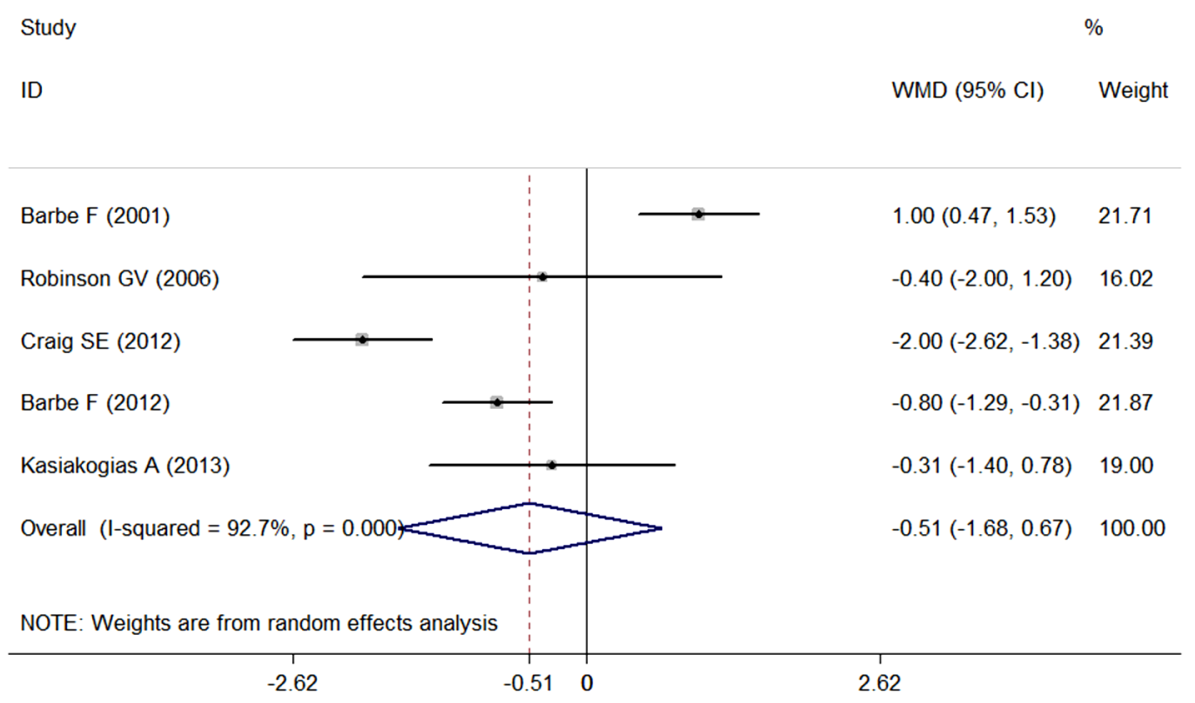

Figure 3 Effect of CPAP on Epworth sleeping scale (ESS). CPAP, continuous positive airway pressure.

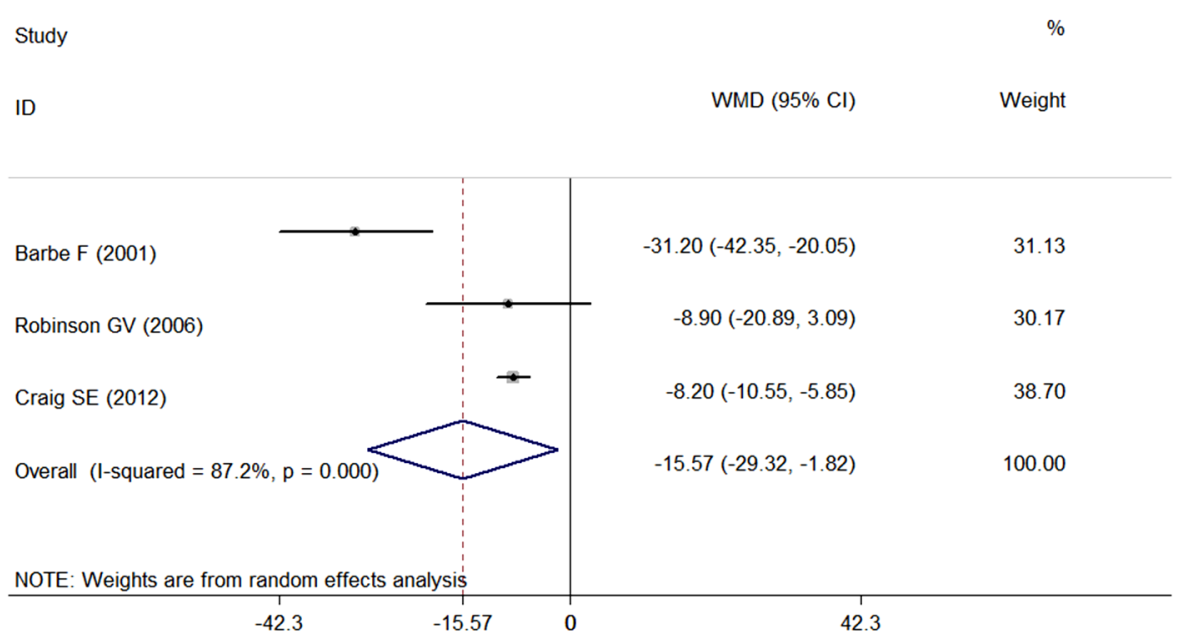

Figure 4 Effect of CPAP on apnea-hypopnea index (AHI) or on oxygen desaturation index (ODI). CPAP, continuous positive airway pressure.

to $0.67 ; \mathrm{P}=0.397)$. Despite the large heterogeneity among studies $\left(\mathrm{I}^{2}=72.7 \%, \mathrm{P}<0.001\right)$, influence analysis (Figure S1) did not find any single study significantly affecting the overall meta-analysis estimate.

There was a strong, statistically significant effect of CPAP on AHI or ODI, reducing it by 15.57 (95\% CI, -29.32 to $-1.82 ; \mathrm{P}=0.026)$ events/h compared with control, which is shown in Figure 4. The result of Barbé et al. (17) caused a large amount of heterogeneity; if this study was removed, the estimate of the treatment effect would be -8.2
(95\% CI, -10.5 to $-5.9 ; \mathrm{P}<0.001)$.

The risk of cardiovascular events was reported in three studies $(8,18,19)$ with 573 patients in the CPAP group and 582 patients in the control group, and the mean follow-up was 3.09 years. The meta-analysis of risk in cardiovascular events is shown in Figure 5; the odds ratio (OR) was 0.80 (95\% CI, 0.50 to $1.26, \mathrm{P}=0.332$ ). In other words, the effect of CPAP on the incidence of cardiovascular events with reference to the control group showed no significant differences. 


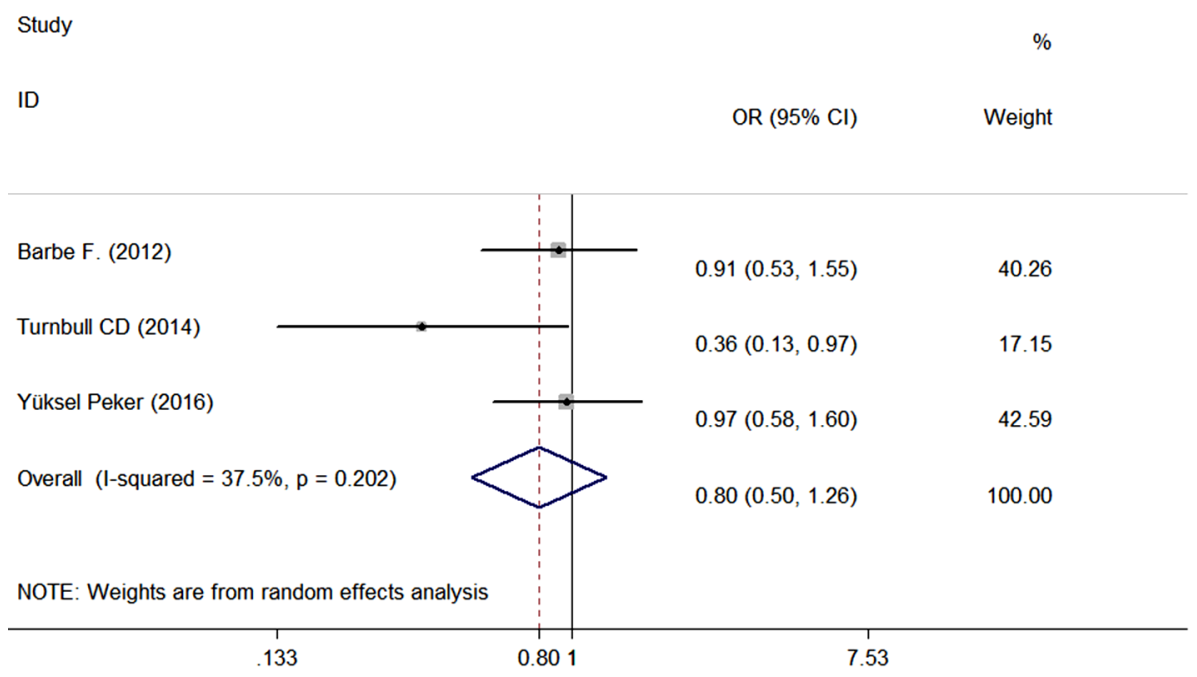

Figure 5 Effect of CPAP on the risk of cardiovascular events (OR). CPAP, continuous positive airway pressure; OR, odds ratio.

Publication bias (Figure S2), influence analysis (Figure S1) and meta-regression (Figure S3) were performed to explain the large degree of heterogeneity among studies. However, this heterogeneity could not be explained by differences in age, gender, BMI, AHI, mean hours of CPAP use, smoking, anti-hypertension drugs, baseline diabetics or the Jadad score. Not one specific study caused the heterogeneity, and there was no published bias either, so we chose the randomeffects model.

\section{Discussion}

The findings of this meta-analysis show that CPAP can reduce OSA severity in non-sleepy patients, and CPAP can minutely reduce the DBP $(-0.92,-1.39$ to $-0.46 \mathrm{mmHg}, \mathrm{P}<0.001)$. However, CPAP seems to have no overall beneficial effect on subjective sleepiness, SBP, or cardiovascular risk. The meta-analysis conducted by Bratton et al. (4) arrived at a similar result, i.e., that DBP was reduced in patients using CPAP $>4 \mathrm{~h} /$ night $(-1.4,95 \% \mathrm{CI},-2.5$ to $-0.4 \mathrm{mmHg}$, $\mathrm{P}=0.008)$. In their meta-analysis, there was also no statistical significance in the change of SBP. Alternative to their paper, we were not able to obtain the full information in the data of the included patients; we relied on the traditional method of abstracting and synthesizing data from published articles. Additionally, we were also able to include a new trial. To date, there are eleven meta-analyses published on the impact of CPAP on BP in patients with OSA (20-30), the result of some of the papers among them show no reduction in SBP and a small reduction in DBP $(21,27)$, which is in accord with our results. However, different from those studies, our analysis was focused on the CPAP effects in non-sleepy OSA patients. Although the reduction in DBP has statistical significance, it is hard to determine the minimal declination less than $1 \mathrm{mmHg}$ that would have clinical importance. According to our findings, non-sleepy OSA patients should generally not be treated with CPAP to reduce BP in clinical practice. Because many patients had normal blood pressure after the antihypertensive drugs at baseline, we speculate a floor effect might exist.

Even though RCTs $(31,32)$ and meta-analyses $(33,34)$ have shown that CPAP could relieve daytime sleepiness in OSA patients, our analysis suggests that CPAP might not further reduce sleepiness in those patients; this result may be due to our study only involving the non-sleepy patients.

OSA is associated with increased risk of mortality and cardiovascular events, and observational studies and RCTs have suggested that CPAP treatment reduces the incidence of mortality and non-fatal cardiovascular events in OSA patients (35-37), especially in those patients with moderate and severe OSA. However, our meta-analysis revealed no significant treatment effect of CPAP on cardiovascular events in nonsleepy OSA patients. A recently published meta-analysis about the effect of CPAP therapy on cardiovascular events (38) had a similar finding that CPAP could not decrease the risk of cardiovascular events compared with the control group (OR, 0.84; 95\% CI, 0.62-1.13; $\mathrm{P}=0.25)$. Considering the limited number of people included and the short duration of follow- 
up, the negative results in our meta-analysis could not exclude the possibility that non-sleepy OSA patients could benefit from long-term CPAP use. Both Barbé et al. (8) and Peker et al. (19) showed a mild beneficial effect of CPAP on cardiovascular events only in patients who used CPAP for $4 \mathrm{~h} /$ night or longer. Maybe favorable adherence to CPAP is required to achieve beneficial effects on cardiovascular outcomes in these patients. Because it is hard to predict patients' compliance by the baseline characteristics (39), analysis of the cost-effectiveness of treatment is needed in non-sleepy OSA patients to assess if there are economic benefits to suggest these patients accept CPAP therapy.

At least so far, we have not obtained enough evidence to strongly support using CPAP in non-sleepy OSA patients in terms of achieving cardiovascular advantage. However, we cannot deny that these patients might receive benefits in other aspects such as reducing inflammation, oxidative stress, sympathetic activity levels, improving quality of life, cognitive function and so on. The MOSAIC trial showed that CPAP can improve vascular endothelial function (9), but CPAP had no effects on cardiac function (10), inflammation markers (12), cholesterol or HbA1c (11). CPAP treatment is really challenging in OSA without daytime sleepiness, and larger randomized studies are needed to identify the association between CPAP treatment and cardiovascular effects in these patients.

Additionally, we should realize that daytime sleepiness is not a specific symptom; many OSA patients do not feel sleepy, especially patients with cardiovascular disease such as chronic heart failure (40) due to the increasing sympathetic activity (41). Evidence has shown that CPAP treatment can greatly improve the left ventricular ejection fraction in the OSA patient with coexisting chronic heart failure (42). In addition, trials reflect that OSA patients tend to underestimate their sleepiness assessed by ESS before CPAP treatment (43). The correlation between the ESS and OSA related variables [AHI or ODI, $\mathrm{SaO}_{2}$, arousal index (ArI)] was weak (44), so only using daytime sleepiness to determine which patient should use CPAP therapy is inappropriate.

\section{Study limitations}

There are several limitations in our meta-analysis. First, only a limited number of patients were in double-blind studies because only two small trials accounting for $6 \%$ patients used sham CPAP in the control group. However, sham CPAP is not the same as an oral placebo tablet; it is not possible to completely blind all patients and staff from real and sham CPAP, and treatment adherence is worse with sham CPAP than with real CPAP (45). In long-term studies, it is now accepted that non-sham controlled trials are the only appropriate and feasible option. Second, the follow-up length of the study varied widely from 1 month to 4.75 years. Third, we did not adjust our analyses for adherence to the CPAP therapy. Fourth, there remained slight differences across all studies in the characteristics of enrolled patients, even though we had broad inclusion criteria. Larger clinical cohorts with more homogeneous populations are needed in the future.

\section{Conclusions}

Even though CPAP therapy can effectively reduce AHI or ODI in non-sleepy OSA patients, there are no overall benefits in cardiovascular events and SBP. CPAP therapy can minimally reduce DBP $(<1 \mathrm{mmHg})$, which may be of little clinical significance. Because evidence of the effects of CPAP therapy in non-sleepy OSA patients is less definitive, larger randomized studies or longer follow-up periods are still needed.

\section{Acknowledgements}

The authors would like to thank Xiaolu Gao for her help in developing the methods for performing the meta-analysis. Funding: This research was supported by National Natural Science Foundation of China (81570085).

\section{Footnote}

Conflicts of Interest: The authors have no conflicts of interest to declare.

\section{References}

1. Franklin KA, Lindberg E. Obstructive sleep apnea is a common disorder in the population-a review on the epidemiology of sleep apnea. J Thorac Dis 2015;7:1311-22.

2. Oksenberg A, Arons E, Nasser K, et al. Severe obstructive sleep apnea: sleepy versus nonsleepy patients. Laryngoscope 2010;120:643-8.

3. Young T, Peppard PE, Gottlieb DJ. Epidemiology of obstructive sleep apnea: a population health perspective. Am J Respir Crit Care Med 2002;165:1217-39.

4. Bratton DJ, Stradling JR, Barbé F, et al. Effect of CPAP on blood pressure in patients with minimally symptomatic obstructive sleep apnoea: a meta-analysis using individual 
patient data from four randomised controlled trials. Thorax 2014;69:1128-35.

5. Moher D, Liberati A, Tetzlaff J, et al. Preferred reporting items for systematic reviews and meta-analyses: the PRISMA statement. Ann Intern Med 2009;151:264-9, W64.

6. Comondore VR, Cheema R, Fox J, et al. The impact of CPAP on cardiovascular biomarkers in minimally symptomatic patients with obstructive sleep apnea: a pilot feasibility randomized crossover trial. Lung 2009;187:17-22.

7. Barbé F, Durán-Cantolla J, Capote F, et al. Longterm effect of continuous positive airway pressure in hypertensive patients with sleep apnea. Am J Respir Crit Care Med 2010;181:718-26.

8. Barbé F, Duran-Cantolla J, Sanchez-de-la-Torre M, et al. Effect of continuous positive airway pressure on the incidence of hypertension and cardiovascular events in nonsleepy patients with obstructive sleep apnea: a randomized controlled trial. JAMA 2012;307:2161-8.

9. Kohler M, Craig S, Pepperell JC, et al. CPAP improves endothelial function in patients with minimally symptomatic OSA: results from a subset study of the MOSAIC trial. Chest 2013;144:896-902.

10. Craig S, Kylintireas I, Kohler M, et al. Effect of CPAP on Cardiac Function in Minimally Symptomatic Patients with OSA: Results from a Subset of the MOSAIC Randomized Trial. J Clin Sleep Med 2015;11:967-73.

11. Craig SE, Kohler M, Nicoll D, et al. Does Continuous Positive Airways Pressure (CPAP) for minimally symptomatic Obstructive Sleep Apnoea (OSA) reduce calculated cardiovascular risk? Primary results from mosaic, a randomized controlled trial. Am J Respir Crit Care Med 2011;183:A2201.

12. Stradling JR, Craig SE, Kohler M, et al. Markers of inflammation: data from the MOSAIC randomised trial of CPAP for minimally symptomatic OSA. Thorax 2015;70:181-2.

13. Craig SE, Kohler M, Nicoll D, et al. Does Continuous Positive Airways Pressure (CPAP) For Minimally Symptomatic Obstructive Sleep Apnoea (OSA) reduce daytime sleepiness? primary results from mosaic, a randomized controlled trial. Am J Respir Crit Care Med 2011;183:950.

14. Robinson GV, Smith DM, Langford BA, et al. Continuous positive airway pressure does not reduce blood pressure in nonsleepy hypertensive OSA patients. Eur Respir J 2006;27:1229-35.

15. Craig SE, Kohler M, Nicoll D, et al. Continuous positive airway pressure improves sleepiness but not calculated vascular risk in patients with minimally symptomatic obstructive sleep apnoea: the MOSAIC randomised controlled trial. Thorax 2012;67:1090-6.

16. Kasiakogias A, Tsioufis C, Thomopoulos C, et al. Effects of continuous positive airway pressure on blood pressure in hypertensive patients with obstructive sleep apnea: a 3-year follow-up. J Hypertens 2013;31:352-60.

17. Barbé F, Mayoralas LR, Duran J, et al. Treatment with continuous positive airway pressure is not effective in patients with sleep apnea but no daytime sleepiness. a randomized, controlled trial. Ann Intern Med 2001;134:1015-23.

18. Turnbull CD, Craig SE, Kohler M, et al. Cardiovascular event rates in the MOSAIC trial: 2-year follow-up data. Thorax 2014;69:950.

19. Peker Y, Glantz H, Eulenburg C, et al. Effect of Positive Airway Pressure on Cardiovascular Outcomes in Coronary Artery Disease Patients with Non-Sleepy Obstructive Sleep Apnea: The RICCADSA Randomized Controlled Trial. Am J Respir Crit Care Med 2016;194:613-20.

20. Liu L, Cao Q, Guo Z, et al. Continuous Positive Airway Pressure in Patients With Obstructive Sleep Apnea and Resistant Hypertension: A Meta-Analysis of Randomized Controlled Trials. J Clin Hypertens (Greenwich) 2016;18:153-8.

21. Hu X, Fan J, Chen S, et al. The role of continuous positive airway pressure in blood pressure control for patients with obstructive sleep apnea and hypertension: a metaanalysis of randomized controlled trials. J Clin Hypertens (Greenwich) 2015;17:215-22.

22. Bratton DJ, Gaisl T, Wons AM, et al. CPAP vs Mandibular Advancement Devices and Blood Pressure in Patients With Obstructive Sleep Apnea: A Systematic Review and Meta-analysis. JAMA 2015;314:2280-93.

23. Iftikhar IH, Valentine CW, Bittencourt LR, et al. Effects of continuous positive airway pressure on blood pressure in patients with resistant hypertension and obstructive sleep apnea: a meta-analysis. J Hypertens 2014;32:234150; discussion 2350.

24. Varounis C, Katsi V, Kallikazaros IE, et al. Effect of CPAP on blood pressure in patients with obstructive sleep apnea and resistant hypertension: a systematic review and metaanalysis. Int J Cardiol 2014;175:195-8.

25. Schein AS, Kerkhoff AC, Coronel CC, et al. Continuous positive airway pressure reduces blood pressure in patients with obstructive sleep apnea; a systematic review and metaanalysis with 1000 patients. J Hypertens 2014;32:1762-73.

26. Montesi SB, Edwards BA, Malhotra A, et al. The effect 
of continuous positive airway pressure treatment on blood pressure: a systematic review and meta-analysis of randomized controlled trials. J Clin Sleep Med 2012;8:587-96.

27. Mo L, He QY. Effect of long-term continuous positive airway pressure ventilation on blood pressure in patients with obstructive sleep apnea hypopnea syndrome: a meta-analysis of clinical trials. Zhonghua Yi Xue Za Zhi 2007;87:1177-80.

28. Haentjens P, Van Meerhaeghe A, Moscariello A, et al. The impact of continuous positive airway pressure on blood pressure in patients with obstructive sleep apnea syndrome: evidence from a meta-analysis of placebo-controlled randomized trials. Arch Intern Med 2007;167:757-64.

29. Alajmi M, Mulgrew AT, Fox J, et al. Impact of continuous positive airway pressure therapy on blood pressure in patients with obstructive sleep apnea hypopnea: a meta-analysis of randomized controlled trials. Lung 2007;185:67-72.

30. Fava C, Dorigoni S, Dalle VF, et al. Effect of CPAP on blood pressure in patients with OSA/hypopnea a systematic review and meta-analysis. Chest 2014;145:762-71.

31. Turnbull CD, Bratton DJ, Craig SE, et al. Predictors of continuous positive airways pressure usage at six months in minimally symptomatic patients. Further data from the mosaic trial. Thorax 2015;70:A20.2-A21.

32. Hardinge FM, Pitson DJ, Stradling JR. Use of the Epworth Sleepiness Scale to demonstrate response to treatment with nasal continuous positive airways pressure in patients with obstructive sleep apnoea. Respir Med 1995;89:617-20.

33. Marshall NS, Barnes M, Travier N, et al. Continuous positive airway pressure reduces daytime sleepiness in mild to moderate obstructive sleep apnoea: a meta-analysis. Thorax 2006;61:430-4.

34. Patel SR, White DP, Malhotra A, et al. Continuous positive airway pressure therapy for treating sleepiness in a diverse population with obstructive sleep apnea: results of a meta-analysis. Arch Intern Med 2003;163:565-71.

35. Nishihata Y, Takata Y, Usui Y, et al. Continuous positive airway pressure treatment improves cardiovascular outcomes in elderly patients with cardiovascular disease and obstructive sleep apnea. Heart Vessels 2015;30:61-9.

36. Martínez-García MA, Campos-Rodríguez F, Catalán-Serra $\mathrm{P}$, et al. Cardiovascular mortality in obstructive sleep apnea in the elderly: role of long-term continuous positive airway pressure treatment: a prospective observational study. Am J Respir Crit Care Med 2012;186:909-16.

37. Doherty LS, Kiely JL, Swan V, et al. Long-term effects of nasal continuous positive airway pressure therapy on cardiovascular outcomes in sleep apnea syndrome. Chest 2005;127:2076-84.

38. Guo J, Sun Y, Xue LJ, et al. Effect of CPAP therapy on cardiovascular events and mortality in patients with obstructive sleep apnea: a meta-analysis. Sleep Breath 2016;20:965-74.

39. Glantz H, Thunström E, Cederin B, et al. Obstructive sleep apnea and adherence to CPAP in coronary artery disease without daytime sleepiness: RICCADSA trial. Scand Cardiovasc J 2010;44:28.

40. Mansfield DR, Gollogly NC, Kaye DM, et al. Controlled trial of continuous positive airway pressure in obstructive sleep apnea and heart failure. Am J Respir Crit Care Med 2004;169:361-6.

41. Taranto Montemurro L, Floras JS, Millar PJ, et al. Inverse relationship of subjective daytime sleepiness to sympathetic activity in patients with heart failure and obstructive sleep apnea. Chest 2012;142:1222-8.

42. Sun H, Shi J, Li M, et al. Impact of continuous positive airway pressure treatment on left ventricular ejection fraction in patients with obstructive sleep apnea: a metaanalysis of randomized controlled trials. PLoS One 2013;8:e62298.

43. Leclerc G, Lacasse Y, Page D, et al. Do obstructive sleep apnea syndrome patients underestimate their daytime symptoms before continuous positive airway pressure treatment. Can Respir J 2014;21:216-20.

44. Kendzerska TB, Smith PM, Brignardello-Petersen R, et al. Evaluation of the measurement properties of the Epworth sleepiness scale: a systematic review. Sleep Med Rev 2014;18:321-31.

45. Djavadkhani Y, Marshall NS, D'Rozario AL, et al. Ethics, consent and blinding: lessons from a placebo/sham controlled CPAP crossover trial. Thorax 2015;70:265-9.

Cite this article as: Zhang D, Luo J, Qiao Y, Xiao Y. Continuous positive airway pressure therapy in non-sleepy patients with obstructive sleep apnea: results of a metaanalysis. J Thorac Dis 2016;8(10):2738-2747. doi: 10.21037/ jtd.2016.09.40 


\section{Search details}

\section{Medline}

$(((((((((($ apnea) $)$ OR OSA) OR OSAS) OR hypopnea) OR obstructive sleep apn*) OR SAHS $))))$ AND $((((($ minimally symptomatic) OR nonsleepy) OR nonsymptomatic) OR asymptomatic) OR non sleepy) OR non symptomatic)) AND ((((cpap) OR continuous positive airway pressure) OR PAP) OR positive pressure) ) AND $((((($ randomized controlled trial[Publication Type]) OR controlled clinical trial[Publication Type]) OR randomized[Title/Abstract]) OR placebo[Title/Abstract]) OR randomly[Title/Abstract]) OR trial[Title]) Filters: Publication date from 1996/01/01 to 2016/04/1

\section{Embase}

\#1. 'apnea'

\#2. OSA

\#3. 'obstructive sleep apnea'

\#4. 'hypopnea'

\#5. SAHS

\#6. OSAHS

\#7. \#1 OR \#2 OR \#3 OR \#4 OR \#5 OR \#6

\#8. 'randomized controlled trial'

\#9. 'controlled clinical trial'

\#10. Randomized: ab

\#11. Placebo: ab

\#12. 'clinical trials as topic'

\#13. randomly: ab

\#14. trial: ti

\#15. \#8 OR \#9 OR \#11 OR \#12 OR \#13 OR \#14

\#16. 'continuous positive airway pressure'

\#17. 'CPAP'

\#18. 'positive airway pressure'

\#19. PAP

\#20. 'positive pressure'

\#21. \#16 OR \#17 OR \#18 OR \#19 OR \#20

\#22. 'minimally symptomatic'

\#23. 'non\$sleepy'

\#24. 'non\$symptomatic'

\#25. 'asymptomatic'

\#26. \#22 OR \#23 OR \#24 OR \# 25’ \#27.'\#7 AND \#15 AND \#21 AND\# 26 AND [1996-2016]/py 

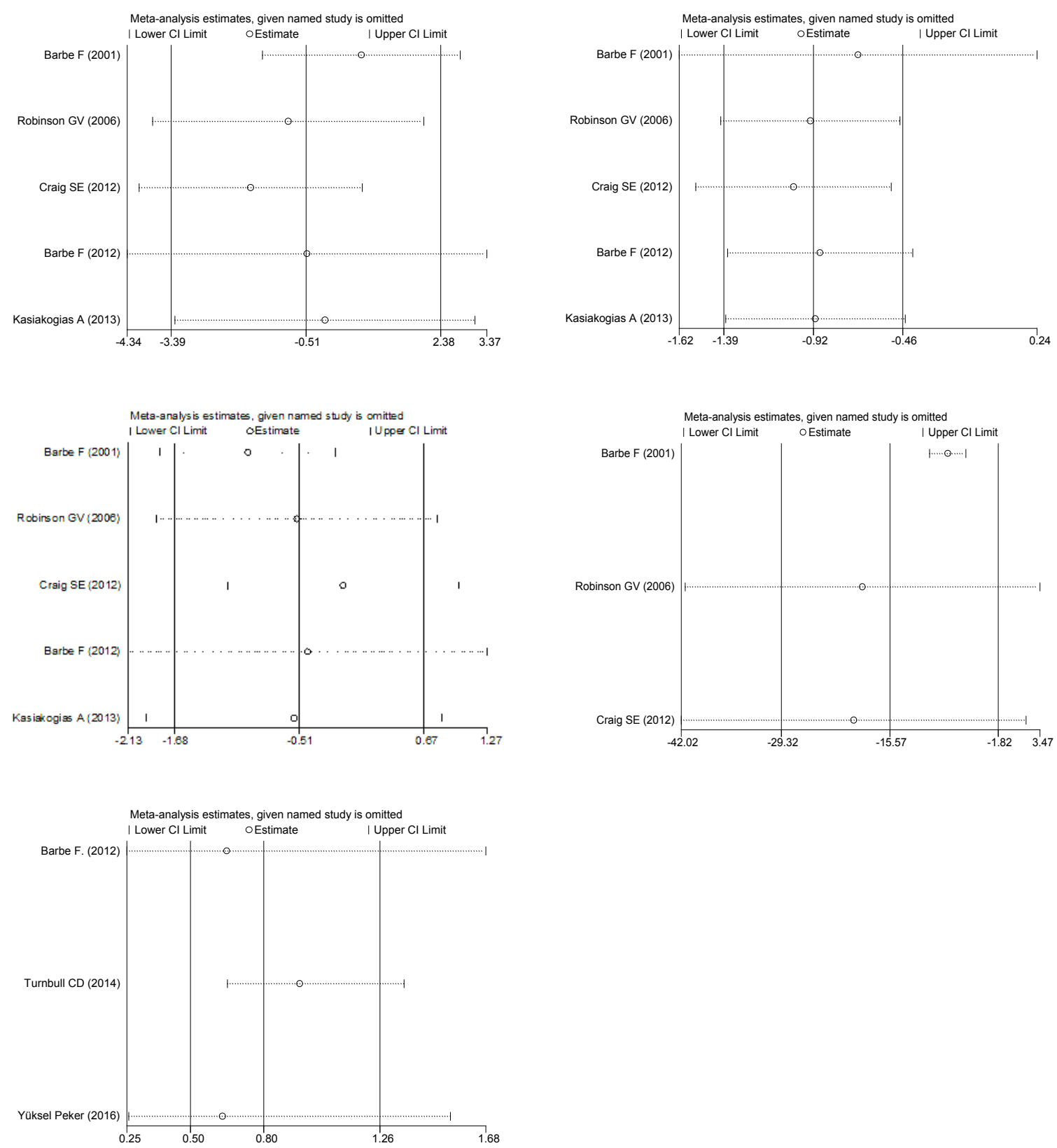

Figure S1 Meta influence analysis in SBP, DBP, ESS, AHI (ODI), and cardiovascular risk. SBP, systolic blood pressure; DBP, diastolic blood pressure; ESS, Epworth sleepiness scale; AHI, apnea-hypopnea index; ODI, oxygen desaturation index. 


\begin{tabular}{|c|c|c|c|c|c|c|c|c|c|}
\hline \multirow[b]{2}{*}{ subgroup | } & \multirow[b]{2}{*}{$\mathrm{n}$} & \multicolumn{2}{|c|}{ Begg's } & \multicolumn{2}{|c|}{ Begg's } & \multicolumn{2}{|c|}{ cont. corr. } & \multicolumn{2}{|c|}{ Egger's } \\
\hline & & score & s.d. & $\mathrm{z}$ & $\mathrm{p}$ & $z$ & $\mathrm{p}$ & bias & $\mathrm{p}$ \\
\hline 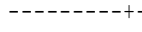 & - & & $-1-1$ & & & & --- & ---- & ----- \\
\hline AHI । & 3 & -1 & 1.915 & -0.52 & 0.602 & 0.00 & 1.000 & -1.45 & 0.651 \\
\hline DBP । & 5 & 0 & 4.082 & 0.00 & 1.000 & -0.24 & 1.000 & -1.01 & 0.557 \\
\hline ESS I & 5 & 0 & 4.082 & 0.00 & 1.000 & -0.24 & 1.000 & 2.56 & 0.391 \\
\hline SBP | & 5 & -2 & 4.082 & -0.49 & 0.624 & 0.24 & 0.806 & -2.01 & 0.480 \\
\hline & & & & & & & & & \\
\hline overall | & 18 & -3 & 7.326 & -0.41 & 0.682 & -0.27 & 0.785 & -0.68 & 0.517 \\
\hline
\end{tabular}

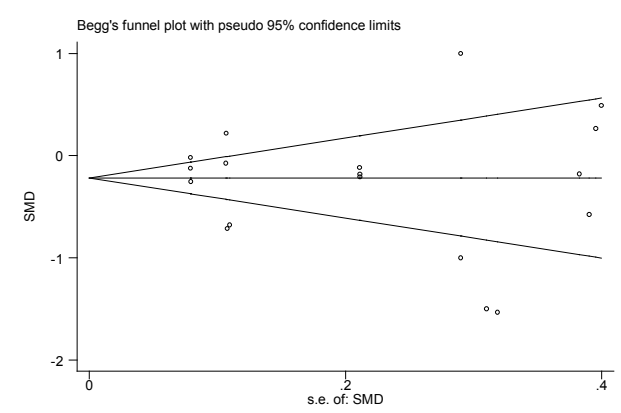

Figure S2 Test for publication bias, Begg's funnel plot and Egger's publication funnel plot.

Meta-regression

REML estimate of between-study variance

o residual variation due to heterogeneity

Proportion of between-study variance explained

With Knapp-Hartung modification

\begin{tabular}{r|rrrrrr}
\hline$\_$ES & Coef. & Std. Err. & $t$ & P > t I & [95\% Conf. Interval] \\
\hline cpap & -.0161243 & .1984937 & -0.08 & 0.937 & -.4583957 & .4261471 \\
cons & -.294497 & .8633343 & -0.34 & 0.740 & -2.218126 & 1.629132 \\
\hline
\end{tabular}

\begin{tabular}{rrr}
$\begin{array}{l}\text { Number of obs } \\
\text { tau2 }\end{array}$ & 12 \\
I-squared_res & $=$ & .5754 \\
Adj R-squared & $=$ & $-12.59 \%$ \\
& \\
\hline [95\% Conf. & Interval $]$ \\
\hline-.4583957 & .4261471 \\
-2.218126 & 1.629132
\end{tabular}

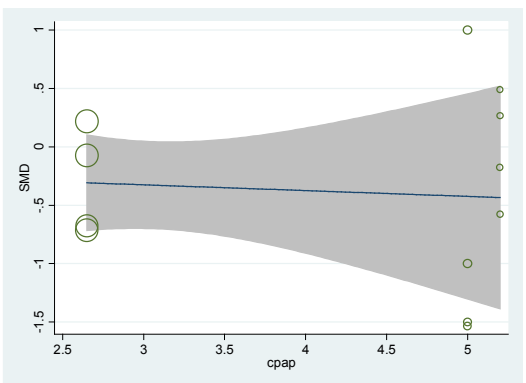

Figure S3 Meta regression-CPAP adherence. CPAP, continue positive airway pressure. 